\title{
RELAÇÃO TERAPÊUTICA COM PACIENTES BORDERLINES NA TERAPIA COMPORTAMENTAL DIALÉTICA
}

THERAPEUTIC RELATIONSHIP WITH BORDERLINE PATIENTS IN DIALECTICAL BEHAVIOR THERAPY

\section{RELACIÓN TERAPÉUTICA CON PACIENTES BORDERLINE EN LA TERAPIA DIALÉCTICA CONDUCTUAL}

\author{
Carine Viegas Cavalheiro* \\ Wilson Vieira Melo ${ }^{* *}$
}

\begin{abstract}
RESUMO
A relação terapêutica tem sido destacada como um dos principais fatores relativos a regulação emocional em pacientes com Transtorno da Personalidade Borderline (TPB). A Terapia Comportamental Dialética (TCD) coloca a relação terapêutica como um de seus pilares terapêuticos devido ao uso da relação como forma de persuasão e modificação de comportamentos desadaptadivos. Diversos estudos têm indicado que esta tem sido uma abordagem eficaz no tratamento do TPB e atualmente é o tratamento de primeira linha de escolha. Este estudo tem como objetivo explorar a relação terapêutica com pacientes borderlines na TCD por meio de uma revisão de literatura. Foram observados fatores essenciais da relação terapêutica como a empatia, proteção, cuidado, flexibilidade, versatilidade, congruência e compromisso. Embora esses fatores devam estar presentes em toda relação terapêutica, no TPB requerem atenção especial. A TCD demonstra articular os fatores da relação terapêutica de modo a contribuir para um maior engajamento do paciente no tratamento.
\end{abstract}

Palavras-chave: Relação terapeuta-paciente. Transtorno de personalidade borderline. Terapia comportamental dialética.

\begin{abstract}
The therapeutic relationship has been highlighted as one of the main factors related to emotional regulation in patients with Borderline Personality Disorder (BPD). The Dialectical Behavior Therapy (DBT) sets the therapeutic relationship as one of its therapeutic pillars due to the use of the relationship as a means of persuasion and change maladaptive behavior. Several studies have indicated that this has been an effective approach in the treatment of BPD and is currently the first-line treatment of choice.
\end{abstract}

\footnotetext{
Texto recebido em 5 de outubro de 2013 e aprovado para publicação em 11 de novembro de 2015.

Psicóloga, servidora técnico-administrativa da UFRGS.E-mail: carineviegas@yahoo.com.br.

* Professor de Pós-Graduação do Instituto WP, psicólogo. E-mail: wilsonmelo1@gmail.com.
} 
This study aims to explore the therapeutic relationship with borderline patients in TCD through a literature reviewed. Observing essential factors of the therapeutic relationship such as empathy, protection, care, flexibility, versatility, consistency and commitment. While these factors must be present in every therapeutic relationship, the TPB require special attention. The TCD demonstrates articulate the factors of the therapeutic relationship in order to contribute to greater patient engagement in treatment.

Keywords: Therapist-patient relation. Borderline personality disorder. Dialectical behavior therapy.

\section{RESUMEN}

La relación terapéutica se ha destacado como uno de los principales factores relacionados con la regulación emocional en los pacientes con trastorno de personalidad borderline (TPB). La terapia dialéctica conductual (TDC) pone la relación terapéutica como uno de sus pilares terapéuticos debido a la utilización de la relación como medio de persuasión y modificación de comportamientos inadaptados. Varios estudios han indicado que éste ha sido un enfoque eficaz en el tratamiento del TPB y actualmente es el tratamiento de primera línea de elección. Este estudio tiene como objetivo explorar la relación terapéutica con los pacientes borderline na TDC a través de una revisión de la literatura. Se observaron factores esenciales de la relación terapéutica como la empatía, la protección, el cuidado, la flexibilidad, versatilidad, congruencia y compromiso. Si bien estos factores deben estar presentes en toda relación terapéutica, en el TPB requiere una atención especial. La TDC demuestra articular los factores de la relación terapéutica con el fin de contribuir a una mayor participación del paciente en el tratamiento.

Palabras clave: Relación terapeuta-paciente. Trastorno límite de la personalidad. Terapia conductual dialéctica.

\section{INTRODUÇÃO}

A relação ou aliança terapêutica consiste em um vínculo colaborativo e emocional entre paciente e terapeuta (Hughes \& Kendall, 2007). É uma façanha, fixada com um propósito no tempo, interativa e que reflete na resposta mútua do paciente para com o terapeuta (Leahy, 2008). Historicamente, a natureza da relação terapêutica tem sido considerada um fator crítico em qualquer forma de psicoterapia bem conduzida. Sua importância começou a ser destacada na abordagem psicanalítica e, desde então, vem ganhando importância por parte dos psicoterapeutas (Scaturo, 2002). 
Por muitos anos, a terapia psicodinâmica teve orgulho dela mesma pelo seu entendimento da relação terapêutica (Leahy, 2008). De acordo com Scaturo (2002), Winnicott já enfatizava a necessidade de a psicoterapia proporcionar um ambiente facilitador para o paciente, em que este possa confiar no seu terapeuta para que consiga ter resultados terapêuticos. Espera-se que o terapeuta consiga desempenhar o papel de um "terapeuta suficientemente bom", que demonstre respeito e preocupação pelas vulnerabilidades trazidas do paciente antes mesmo de se pensar em técnicas terapêuticas mais específicas.

Embora as explicações psicanalíticas tenham sido de considerável importância no entendimento da relação terapêutica, na prática, mostram-se, frequentemente, um tanto obscuras e difíceis de serem utilizadas (Leahy, 2008). Scaturo (2002) destaca a contribuição da abordagem humanística na ênfase da relação terapêutica. Desse modo, Rogers destacou três condições facilitadoras na relação cliente-terapeuta: a genuinidade, a empatia e o respeito. A genuinidade refere-se ao terapeuta demonstrar congruência, sinceridade e honestidade ao paciente. A empatia tem relação com o entendimento verdadeiro da realidade subjetiva do paciente. $\mathrm{E}$, por último, o respeito, relacionado à necessidade do terapeuta de conceber qualquer comportamento do paciente com consideração.

Já abordagem comportamental, historicamente teve uma postura mais cética com a relação terapêutica, não a colocando em uma posição de destaque em sua teoria. Em contrapartida, embora a Terapia Cognitivo-Comportamental (TCC) tenha sido bastante criticada, acusada por ignorar o papel da relação terapêutica, há muitas dimensões para a formulação de caso e intervenção que sugerem que essa abordagem pode ser um forte modelo teórico para o entendimento dos impasses que surgem na psicoterapia (Leahy, 2008; Scaturo, 2002).

Durante a última década, o interesse pela relação terapêutica na TCC tem se expandido (Leahy, 2008). Hughes e Kendall (2007) em uma pesquisa sobre as consequências da relação terapêutica no tratamento de crianças com diagnóstico de transtornos de ansiedade, na abordagem cognitivo-comportamental, concluíram que crianças que apresentam uma boa relação terapêutica com seus terapeutas tendem a estar mais comprometidas no tratamento e mais dispostas a realizar as tarefas terapêuticas propostas. A Terapia Comportamental Dialética (TCD) é uma das principais abordagens da chamada Terceira Onda em Terapia Cognitiva, em que a relação terapêutica recebe especial atenção no tratamento de pacientes. As abordagens da Terceira Onda costumam ter um caráter integrativo nas suas intervenções, muitas delas focadas no relacionamento terapeutapaciente, de modo que a própria relação terapêutica pode ser entendida como uma ferramenta de reestruturação cognitiva, regulação emocional e também modificação comportamental (Melo, Sardinha \& Levitan, 2014). 
Langhoff, Baer, Zubraegel e Linden (2008) também pesquisaram a relação terapêutica na TCC, sob a perspectiva do paciente, do terapeuta e de um terceiro, em indivíduos com transtornos de ansiedade, mas utilizando uma amostra de adultos. Os resultados mostraram altos escores positivos em todos os aspectos da escala VAS (Fähndrich \& Linden, 1982), que mede a qualidade da aliança terapêutica. Dessa forma, tanto paciente quanto terapeuta e observador descreveram ter encontrado nas sessões altos índices de empatia, transparência, foco, estrutura, progresso e cooperação.

A aliança terapêutica vem sendo positivamente relacionada aos resultados do tratamento e pode, inclusive, por si só, levar a mudanças terapêuticas (Langhoff et al., 2008). La Rie, Noordenbos, Donker e Furth (2008) investigaram a qualidade do tratamento de transtornos alimentares sob a perspectiva do terapeuta e do paciente e verificaram que, embora ambos tenham avaliado alguns tópicos diferentemente, os dois consideraram de suma importância para a qualidade do tratamento, entre outros fatores, a aliança terapêutica e as habilidades de comunicação. Além disso, observaram que os pacientes, mais que os terapeutas, destacaram a importância da relação com o terapeuta para o sucesso da terapia.

Percebe-se que a relação terapêutica é um componente central no sucesso de uma psicoterapia (Howard, Turner, Olkin \& Mohr, 2006). Entretanto parece que nem sempre ela se constitui uma tarefa fácil, principalmente em determinadas psicopatologias, tal como o Transtorno da Personalidade Borderline (TPB).

O TPB é uma psicopatologia caracterizada por um persistente padrão de instabilidade dos afetos, da identidade, dos relacionamentos interpessoais e excessiva impulsividade. Tais características se estruturam na personalidade do indivíduo no início da vida adulta e apresenta-se em diversos contextos. Os critérios diagnósticos incluem sintomas, como instabilidade afetiva, sentimentos crônicos de vazio, comportamentos para evitar um abandono real ou imaginário, relacionamentos intensos e instáveis, perturbação da identidade, impulsividade; comportamento automutilante ou suicida, entre outros (American Psychiatric Association [APA], 2014).

A causa do TPB é complexa e engloba diferentes fatores que se interrelacionam (Lieb, Zanarini, Schmahl, Linehan \& Bohus, 2004). A TCD (modelo de referência para o tratamento do TPB) aponta que a etiologia do transtorno está relacionada a uma disfunção do sistema de regulação emocional, proveniente de irregularidades biológicas e de ambientes invalidantes na infância, que desconsideram e rejeitam as respostas emocionais dos indivíduos (Linehan, 2010). 
A interação de fatores genéticos e experiências aversivas na infância, como negligência e abuso, ocasionam uma desregulação emocional e impulsividade, que, por sua vez, levam a desregulação comportamental e conflitos psicossociais, que novamente reforçam a desregulação emocional e a impulsividade, em um ciclo vicioso. Dessa forma, a desregulação emocional faz com que o indivíduo borderline apresente disfunções em quatro grandes áreas: afetiva, cognitiva, comportamental e, como consequência, nos relacionamentos interpessoais (Lieb et al., 2004).

A relação terapêutica é uma das principais prioridades do tratamento do TPB sob a perspectiva da TCD (Blennerhassett \& O'raghallaigh, 2005). Essa abordagem foi desenvolvida pela psicóloga Marsha Linehan e é considerada um tipo de terapia cognitivo-comportamental que emprega diversas estratégias baseadas em técnicas de aceitação, métodos e conceitos da ciência comportamental, práticas incorporadas da prática zen e outros métodos contemplativos, tais como mindfulness (McMain, Korman \& Dimeff, 2001), com o intuito de viabilizar um tratamento mais efetivo para indivíduos com grave desregulação emocional, como as observadas no diagnóstico de TPB (Blennerhassett \& O'raghallaigh, 2005). Desde então, pesquisas vêm sendo realizadas demonstrando sua eficácia (Kröger et al., 2010; Neacsiu, Rizvi \& Linehan, 2010). A dialética fundamental nessa abordagem é entre validação e aceitação dos pacientes como eles são, ao mesmo tempo em que se auxilia na mudança do que se tornaram. O objetivo central é ajudar na regulação emocional e no desenvolvimento de habilidades ensinadas na terapia (Blennerhassett \& O'raghallaigh, 2005).

Devido às características do TPB, a relação terapêutica é permeada por particularidades que recebem especial atenção dentro da abordagem da TCD (Melo \& Fava, 2012). A mudança de comportamentos do paciente que possa interferir na terapia é uma das metas primárias de tratamento dentro da TCD (Linehan, 2010). Assim, o objetivo deste estudo foi explorar e discutir a relação terapêutica com pacientes borderline sob a perspectiva da TCD.

\section{METODOLOGIA}

A pesquisa bibliográfica foi realizada no portal de periódicos CAPES, utilizando palavras-chave como therapeutic relationship, borderline personality disorder, cognitive-behavioral therapy e dialectical behavior therapy, bem como seus respectivos termos traduzidos para o português. Pesquisaram-se também os artigos citados nas referências dos textos encontrados e obras clássicas da área, em inglês e português. 


\section{RESULTADOS}

Diversos fatores são importantes para que a relação terapêutica se estabeleça, entre eles se encontram a empatia, a proteção, o cuidado, a flexibilidade, a versatilidade, a congruência e o compromisso (Howard et al., 2006; Leahy, 2008).

\subsection{Empatia}

A empatia, descrita como a habilidade do terapeuta em sintonizar-se com as emoções vivenciadas pelo paciente e ainda conseguir atribuir significados a elas (Thwaites \& Levy, 2007), pode ser considerada um dos elementos centrais na relação terapêutica. De uma maneira geral, a empatia é um fator importante que deve estar presente no tratamento de qualquer transtorno (Scaturo, 2002), entretanto no TPB ela pode apresentar certas particularidades.

Sabe-se que os pacientes borderlines apresentam um déficit no aprendizado das emoções decorrentes do ambiente invalidante presente em sua infância (Lynch, Trost, Salsman \& Linehan, 2007). Isso faz com que neguem as expressões emocionais dos sujeitos e interpretem suas próprias experiências emocionais equivocadamente (McMain, Korman \& Dimeff, 2001). Dessa forma, muitas vezes não se permitem aprender a entender, rotular, regular ou até mesmo tolerar respostas emocionais, nem a resolver problemas. Em vez disso, podem oscilar entre a inibição emocional e a extrema labilidade emocional (Crowell, Beauchaine \& Linehan, 2009).

Dessa forma, no tratamento do TPB, o terapeuta encontra um sujeito extremamente vulnerável, que pode não ter aprendido a confiar em seus próprios sentimentos como respostas acuradas e legítimas dos eventos que o cercam. Com frequência, indivíduos com o diagnóstico de TPB invalidam suas próprias percepções, adotando as características do ambiente invalidante e vasculhando o ambiente em busca de pistas de como reagir a essas interpretações (Melo, 2014). Assim, podem se tornar um indivíduo com crenças de que suas experiências são resultado de traços de características inaceitáveis e indesejáveis socialmente. Devido a essa instabilidade e desregulação emocional, podem entender que são maus por sentirem raiva, que são preguiçosos por não resolverem as tarefas rapidamente ou ainda que são fracos por sentirem medo. Tais interpretações acabam se confirmando conforme suas experiências de vida acabam sendo interpretadas sistematicamente como resultantes desse padrão de desregulação comportamental, cognitiva e interpessoal (Melo, 2014). Devido a essa instabilidade emocional, aprenderam que expressões negativas de afeto 
não são toleradas e que, em vez disso, são interpretadas como características que devem ser ignoradas ou passíveis de punição. Percebem-se como fatalmente defeituosos, sentindo ódio de si mesmos, desgosto e vergonha (Linehan, 2010; McMain et al., 2001).

Nesse contexto, a TCD enfatiza que o terapeuta deve ser empático e validar os sentimentos do paciente (Blennerhassett \& O'raghallaigh, 2005), mostrando que o compreende e que está tentando ajudá-lo a entender que suas respostas emocionais fazem sentido (McMain et al., 2001).

Muitas vezes, essa tarefa é árdua, pois exige uma sensibilidade muito grande do terapeuta para perceber o que o paciente está necessitando em cada momento (Leahy, 2008). Validar as emoções do paciente não equivale a aprovar o comportamento dele (Melo, 2014). Assim, para desenvolver tal característica, deve antes ter conseguido a capacidade de ser empático.

O termo dialética pressupóes equilíbrio (Linehan, 2010). Nesse contexto, validação (estratégias de aceitação) e mudança (estratégias de resolução de problemas) devem caminhar juntas no processo psicoterápico. Um problema que costuma ocorrer no tratamento desse tipo de psicopatologia é a tendência de alguns terapeutas em priorizar somente as estratégias de mudança em detrimento das estratégias de validação. Embora aquelas sejam de suma importância no tratamento de pacientes borderlines, o risco que se corre em destacar somente as estratégias de mudança é recapitular os aspectos do ambiente invalidante (McMain et al., 2001). Um paciente que está bastante embotado, por exemplo, pode interpretar as estratégias para a mudança como uma invalidação, podendo entender que o terapeuta está ignorando o seu real sofrimento (Leahy, 2008).

Assim, para que o terapeuta consiga estabelecer um plano terapêutico adequado para o paciente, deve antes ter a sensibilidade de conhecer o sofrimento deste, sendo empático com seus sentimentos. Dessa forma, especialmente com os pacientes borderlines, uma das maneiras de demonstrar a empatia pode ser a validação dos sentimentos destes, negligenciados na infância.

\subsection{Proteção e cuidado}

Além da empatia, outros elementos importantes para a relação terapêutica são a proteção e o cuidado. Os pacientes, de um modo geral, esperam que seus terapeutas se mostrem como figuras cuidadosas e de proteção, que demonstrem preocupação, principalmente nos casos em que eles tiveram esses atributos negligenciados na sua história de vida (Scaturo, 2002). 
Entretanto o nível de cuidado que, muitas vezes, o terapeuta tem de despender com os pacientes borderlines parece tomar maiores proporçôes que em outros transtornos mentais, em razão da grande vulnerabilidade emocional presente. Essa desregulação afetiva manifesta-se pela elevada sensibilidade e reatividade a estímulos emocionais e a um lento retorno para um nível emocional basal (Crowell et al., 2009; Lynch, Chapman, Rosenthal, Kuo \& Linehan, 2006), decorrentes do aprendizado com o ambiente invalidante, o qual somente dava atenção para as reações emocionais mais intensas do sujeito, reforçando ainda mais seus comportamentos extremos. Imerso nesse ambiente, o indivíduo aprendeu que somente o extremo pode efetuar respostas no ambiente (McMain et al., 2001). Assim, os pacientes com TPB apresentam dificuldade de regular todo o seu repertório de respostas associado aos estados emocionais (Crowell et al., 2009; Lynch et al., 2006). É comum ocorrerem intensos afetos disfóricos, como raiva, tristeza, vergonha, pânico, terror e sentimentos crônicos de vazio e solidão. Frequentemente mudam rapidamente de uma reação emocional a outra, podendo vivenciar diversos estados emocionais negativos e períodos de eutimia em apenas um dia (Lieb et al., 2004). As mudanças emocionais parecem exageradas e imprevisíveis (Cole, Llera \& Pemberton, 2009). A teoria biossocial, que fundamenta a TCD, pressupõe que tais características são oriundas da interação do ambiente com predisposições biológicas, tais como hiperatividade do sistema límbico, bem como um pobre controle de impulsos associados a áreas específicas do córtex pré-frontal, que, em contato com um ambiente de profunda invalidação, levam à desregulação emocional presente no TPB. ${ }^{1}$ Além disso, estudos apontam que familiares de pacientes Borderlines apresentam cerca de cinco vezes mais chances de desenvolverem o transtorno, o que indica uma influência genética provavelmente associada à gênese do quadro clínico (APA, 2014).

Assim, comportamentos que, na terapia, são concebidos com naturalidade pela maioria dos pacientes, para estes podem ser vivenciados como aniquiladores. As férias do terapeuta, por exemplo, podem ser interpretadas como mais uma situação de abandono na vida deles e, consequentemente, fazem com que se manifeste uma série de comportamentos desadaptativos. Dessa forma, no protocolo de tratamento da TCD, essas situações já devem ser previstas pelo terapeuta e antecipadamente trabalhadas em sessão (Linehan, 2010).

A sensação do terapeuta é de estar muitas vezes "pisando em ovos", tamanha a vulnerabilidade do paciente borderline (Linehan, 2010). Assim como pacientes vítimas de queimaduras na pele, pacientes borderline frequentemente funcionam como se o menor movimento pudesse resultar em uma insuportável

Para uma revisão completa, ver Linehan (2010). 
dor emocional, que leva à perda do controle afetivo, cognitivo, interpessoal e comportamental. Linehan (2010) compara tais características de vulnerabilidade emocional a queimaduras emocionais. É como se fossem fóbicos a todos os sinais associados às emoções negativas e, dessa forma, tentam escapar dessas pistas com evitação ou agressão (McMain et al., 2001).

Dessa forma, a conduta cuidadosa, por parte do terapeuta, que deve fazer parte de qualquer relação terapêutica, no caso de pacientes borderlines, toma proporções muito maiores e exige uma acentuada atenção. Uma das estratégias comumente adotadas na TCD é a utilização de ligações telefônicas para manejo de situações de crise (Blennerhassett \& O'raghallaigh, 2005). As ligações têm o intuito de auxiliar o paciente a enfrentar as dificuldades, reforçando a utilização de habilidades trabalhadas nas sessões e incentivar a permanência no tratamento. McQuillan et al. (2005) destacaram esse tipo de abordagem em um estudo com pacientes borderlines em situação de crise e observaram que o tratamento foi bem tolerado pelos pacientes, sendo que $82 \%$ conseguiram manter-se em todo o tratamento e apenas $6 \%$ necessitaram de internação psiquiátrica. Além disso, encontram significante melhora nos índices de desesperança e depressão. Esses dados apontam para a importância da proteção e do cuidado com pacientes borderlines no manejo da sintomatologia e na adesão ao tratamento, uma das principais dificuldades no tratamento desses pacientes.

\subsection{Flexibilidade e versatilidade}

É importante que a terapia, de um modo geral, constitua-se em um ambiente de flexibilidade e versatilidade, para que se possa discutir e resolver os impasses que surgem no decorrer do tratamento (Scaturo, 2002). No tratamento do TPB, a característica de flexibilidade se faz imprescindível (Linehan, 2010).

Uma das peculiaridades do TPB é a perturbação cognitiva. É comum os pacientes com TPB apresentarem rigidez cognitiva e pensamento dicotômico (Linehan, 2010). Além disso, podem apresentar ideias supervalorizadas acerca do fato de se perceberem como "maus", de desconfiança, além de ideias de referência (Lieb et al., 2004). Assim, conflitos que para alguns pacientes poderiam ser resolvidos com naturalidade e tranquilidade, para eles podem despender mais tempo e energia (Linehan, 2010). Dessa forma, o terapeuta necessita ser ainda mais flexível e versátil para conseguir manejar os impasses que emanam na sessão.

Pacientes borderlines costumam apresentar dificuldade na resolução de problemas e acabam, por vezes, simplificando-os demasiadamente (Melo \& Fava, 2012). Podem, por exemplo, acreditar que conseguem parar de realizar 
os comportamentos automutilatórios pela simples força de vontade e que, assim, não necessitam praticar tarefas de resolução de problemas para prevenir a recorrência desses comportamentos disfuncionais (McMain et al., 2001). Dessa forma, na TCD, cabe ao terapeuta ser flexível e manejar essa situação de forma que consiga mostrar ao paciente que entende sua dificuldade, ao mesmo tempo em que o ajuda com estratégias de mudança (Trupin, Steward, Beach \& Boesky, 2002).

Entretanto se deve tomar cuidado com a característica de passividade ativa presente nesse transtorno, ou seja, o padrão de abordar os problemas passiva e impotentemente, pois, muitas vezes, mobilizam o ambiente e o terapeuta para resolvê-los por ele (McMain et al., 2001). Como uma das estratégias terapêuticas na TCD é ensinar habilidades em áreas em que há déficits (Neacsiu et al., 2010), o terapeuta, deve então, com flexibilidade, estar atento para essa estratégia do paciente e ensinar as habilidades para resolver o problema, e não os resolver por ele.

Muitos pacientes chegam à terapia com a queixa de apresentarem dificuldade nas relações interpessoais ou então trazem isso como algo secundário (Melo \& Fava, 2012). Tais pacientes, muito provavelmente, mostrarão essas dificuldades na relação terapêutica. No caso de pacientes borderlines, a perturbação nos relacionamentos interpessoais, constitui-se em uma das principais dificuldades. Esses sujeitos costumam envolver-se em relacionamentos instáveis e intensos (Lieb et al., 2004) e apresentam comportamentos que dificultam o estabelecimento e a manutenção dessas relações (Cole et al., 2009), inclusive a relação terapêutica.

O profundo medo dos pacientes borderline de serem abandonados faz com que busquem, de todas as formas, não ser deixados sozinhos. Dessa forma, podem ligar repetidamente para as pessoas ou ficar próximos em demasia dos que estão a seu redor, sendo considerados, por vezes, como "pegajosos" (Lieb et al., 2004). Na sessão, podem, por exemplo, realizar frenéticos esforços para agradar a seu terapeuta, ligar demasiadamente para ele e demandar sessóes extras sem que haja realmente necessidade para tal fato. Em contraponto ao carinho intenso, podem surgir afetos negativos em demasia, desproporcionais à situação, como consequência da dificuldade de regulação emocional (McMain et al., 2001), principalmente quando se sentem rejeitados ou percebem indiferença, negligência ou omissão por parte da outra pessoa (APA, 2014), como no caso do terapeuta. Não raro, por exemplo, decidem encerrar precocemente o tratamento ou apresentam uma conduta agressiva (Leahy, 2008). 
Pode também ocorrer o outro oposto, a supressão emocional, como consequência da dificuldade de regulação emocional e pelo padrão de evitação dos sinais de emoção negativa (Melo \& Fava, 2012). Na terapia, ela pode aparecer por meio de comportamentos de dissociação durante a sessão (McMain et al., 2001), como em casos em que o sujeito apresenta um supercontrole da raiva, mostrando-se passivos e submissos em situaçôes em que deveriam expressar seus sentimentos (Linehan, 2010). Podem, por exemplo, em uma situação em que sentem raiva, ficar em silêncio e expressar uma rigidez facial. Quando isso acontece, é muito difícil para o terapeuta identificar o que o paciente está sentindo (McMain et al., 2001).

Dessa forma, a relação terapêutica parece configurar-se em uma montanha russa de expressões emocionais. O paciente pode subir ao topo máximo das emoções, demonstrando intenso carinho ou intensa raiva, como pode cair para o total desprendimento sentimental. Pacientes borderline, em razão da intensidade emocional e respostas imprevisíveis, costumam terminar, de forma turbulenta, seus relacionamentos e apresentar estratégias mal adaptativas que, muitas vezes, provocam raiva e receio nos outros (Lieb et al., 2004). Essa constelação de reações emocionais pode provocar no terapeuta sentimentos negativos, como raiva e medo, que podem, até mesmo, fazê-lo desistir de tratar esse paciente.

É importante que o terapeuta esteja atento e procure auxílio como supervisão clínica, tanto em grupos quanto individualmente, quando perceber tais reações emocionais. Lynch et al. (2007) apontam que um dos critérios da TCD para o tratamento de pacientes borderline é a participação do terapeuta em grupo de supervisão clínica. Dessa forma, além de ter flexibilidade com o paciente, deve ser flexível consigo mesmo e reconhecer que necessita de auxílio. Essas dificuldades na relação terapêutica podem ser úteis para o tratamento do paciente quando bem conduzidas. Podem servir de janela para outros relacionamentos, por permitir que se conheça como o paciente costuma comportar-se e, assim, ajudá-los nas estratégias de mudança (Leahy, 2008).

\subsection{Congruência e compromisso}

A estrutura fornecida pela TCD, com objetivos de tratamento bem delimitados e coerência teórica, contribui para a efetividade no tratamento do TPB (Melo, 2014). Entretanto para isso é importante que terapeuta e paciente estejam de acordo em relação aos objetivos do tratamento, concordem em relação à origem das dificuldades do paciente e com a forma de tratamento que será utilizada (McQuillan et al., 2005). O descaso do terapeuta e paciente em 
delimitar o que deve ser priorizado na terapia pode ser considerado, por si só, uma invalidação, pois remete novamente a um ambiente que não reconhece as demandas emocionais do sujeito (McMain et al., 2001).

Outro elemento fundamental para o sucesso do tratamento na TCD é o compromisso do terapeuta e do paciente para com a terapia. Ambos devem estar comprometidos e engajados no tratamento, para que este possa ser viabilizado (Howard et al., 2006). Indivíduos borderlines costumam apresentar intensa impulsividade que pode manifestar-se por meio de comportamentos de risco (Cole et al., 2009), como comportamentos suicidas ou parassuicidas, abuso de substâncias, distúrbios alimentares, entre outros (McMain et al., 2001). Dessa forma, na TCD, terapeuta e paciente devem comprometer-se com uma das prioridades iniciais do tratamento, que é eliminar os comportamentos de risco (Lynch et al., 2007), os que atrapalham a terapia e aqueles que interferem na qualidade de vida, ao mesmo tempo em que estimula habilidades de resolução de problemas (Melo, 2014). Esses cuidados parecem contribuir na melhora significativa da sintomatologia do TPB e no aumento do funcionamento global dos pacientes (James, Taylor, Winmill \& Alfoadari, 2008).

O terapeuta na TCD deve estar atento à competência aparente, usualmente presente no TPB. Esses indivíduos, por vezes, mostram incongruência entre o que expressam verbalmente e o que fica no nível não verbal. Um paciente pode, por exemplo, em uma sessão de grupo, participar demonstrando confiança e pouca ansiedade e vergonha, quando, na verdade, está sofrendo. Dessa forma, como não comunica o que realmente está sentindo e esquiva-se de pedir ajuda ou a pede de maneira inadequada, seu afeto intenso pode desencadear comportamentos disfuncionais, como automutilação e abuso de substância (McMain et al., 2001).

Além das características prototípicas dos pacientes borderlines, as reações que a configuração sintomatológica desperta no terapeuta também podem prejudicar o compromisso deste em ajudar tais pacientes. Como já salientado anteriormente, a perturbação emocional presente nesses indivíduos propiciam uma cascata emocional e, em razão disso, alterações comportamentais e cognitivas, que, muitas vezes, constituem-se uma carga demasiadamente pesada para as pessoas que estão a seu redor (Linehan, 2010). No caso da psicoterapia, quem mais sente essa intensidade afetiva é o próprio terapeuta.

Dessa forma, faz-se imprescindível o terapeuta participar semanalmente de reuniōes de equipe, estudar manuais de tratamento, participar de supervisões e estar sempre buscando mais informaçóes. Isso proporciona um aumento da capacidade do terapeuta em retomar a motivação e seu compromisso, realizando, assim, um tratamento mais efetivo (Lynch et al., 2007). 


\section{DISCUSSÃO E CONCLUSÃO}

Não por acaso, a TCD coloca a relação terapêutica como uma de suas principais estratégias de tratamento. Sem trabalhar o vínculo, os demais objetivos terapêuticos acabam ficando comprometidos e difíceis de serem atingidos. A TCD vem apresentando resultados satisfatórios no tratamento do TPB (Linehan, 2010; Melo, 2014). Entretanto dificuldades ainda persistem, principalmente relacionadas ao impacto que esses pacientes proporcionam a seus terapeutas. A relação terapêutica ocupa papel de destaque nas diferentes abordagens psicoterápicas. Esse fenômeno é importante no tratamento de qualquer de transtorno, mas, em alguns, como no caso do TPB, a relação terapêutica apresenta algumas particularidades. A TCD coloca a relação terapêutica como uma das principais estratégias de tratamento, posto que o TPB se caracteriza por um padrão de instabilidade afetiva, cognitiva e comportamental, e acentuada impulsividade. Comportamentos automutilatórios, relações instáveis, sentimentos crônicos de vazio, perturbação da identidade, entre outros sintomas, fazem parte da configuração do transtorno (APA, 2014) e merecem especial atenção dentro do tratamento psicoterápico. A TCD aponta que o TPB é consequência de predisposição biológica aliada a um ambiente invalidante na infância, que negligenciou as experiências emocionais dos indivíduos e, dessa forma, não os ensinou a rotular, a regular e a expressar suas emoções (Melo, 2014). Assim, os sintomas presentes nessa psicopatologia estão vinculados à dificuldade em lidar com as emoções.

Ocorre, muitas vezes, de o terapeuta encontrar dificuldades consideráveis no tratamento do paciente com TPB em função da intensidade e instabilidade emocional usualmente presentes no transtorno. Empatia, proteção, cuidado, flexibilidade, versatilidade, congruência e compromisso, fatores essenciais de uma positiva relação terapêutica, devem ser ainda mais destacados no tratamento do TPB.

O terapeuta, dessa forma, deve ser empático com o sofrimento do paciente, mostrar que o entende e, ao mesmo tempo, validar suas experiências emocionais. Deve mostrar-se como uma figura de proteção e cuidado diante de suas oscilações afetivas. Ser flexível e versátil perante sua rigidez cognitiva, suas dificuldades de resolução de problemas e de relacionamento, mostrando que as entende ao mesmo tempo em que trabalha estratégias de mudança. Deve buscar um consenso com o paciente, a congruência com os objetivos do tratamento e com as atividades propostas, bem como o comprometimento com o tratamento, atentando-se às dificuldades que possivelmente serão encontradas. 
Embora a TCD coloque a relação terapêutica como um de seus pilares no tratamento do TPB, observa-se que a maior parte das pesquisas, embora apontem aspectos da relação terapêutica como primordiais no tratamento, aborda com maior ênfase a aquisição de habilidades. Percebe-se uma escassez de estudos que tenham o objetivo de avaliar a relação terapêutica no TPB na perspectiva na TCD. Esses dados são essenciais para o aprimoramento do tratamento do TPB, possibilitando maior adesão do paciente ao tratamento e proporcionando maior suporte ao terapeuta. 


\section{REFERÊNCIAS}

American Psychiatric Association. (2014). Manual diagnóstico e estatístico dos transtornos mentais: DSM-5 (5a. ed.). Porto Alegre: Artmed.

Blennerhassett, R. C. \& O'raghallaigh, J. W. (2005). Dialectical behaviour therapy in the treatment of Borderline Personality Disorder. British Journal of Psychiatry, 186, 278-280. Recuperado a partir de http://bjp.rcpsych.org/ content/bjprcpsych/186/4/278.full.pdf

Cole, P. M., Llera, S. J. \& Pemberton, C. K. (2009). Emotional instability, poor emotional awareness, and the development of borderline personality. Development and Psychopathology, 21(4), 1293-1310. Recuperado a partir de https://doi.org/10.1017/S0954579409990162

Crowell, S. E., Beauchaine, T. P. \& Linehan, M. M. (2009). A biosocial developmental model of borderline personality: elaborating and extending Linehan's Theory. Psychol Bull. 135(3), 495-510. Recuperado a partir de https://doi.org/10.1037/a0015616

Fähndrich, E. \& Linden, M. (1982). Zur reliabilität und validität der stimmungsmessung mit der visuellen analog-skala (VAS). Pharmacopsychiatria, 15, 90-94.

James, A. C., Taylor, A., Winmill, L. \& Alfoadari, K. (2008). A preliminary community study of dialectical behaviour therapy (DBT) with adolescent females demonstrating persistent, deliberate self-harm (DSH). Child and Adolescent Mental Health, 13(3), 148-152. Recuperado a partir de http:// onlinelibrary.wiley.com/doi/10.1111/j.1475-3588.2007.00470.x/abstract

Howard, I., Turner, R., Olkin, R. \& Mohr, D. C. (2006). Therapeutic alliance mediates the relationship between interpersonal problems and depression outcome in a cohort of multiple sclerosis patients. Journal of Clinical Psychology, 62(9), 1197-1204. Recuperado a partir de https://doi.org/10.1002/jclp.20274

Hughes, A. A. \& Kendall, P. C. (2007). Prediction of cognitive behavior treatment outcome for children with anxiety disorders: therapeutic relationship and homework compliance. Behavioural and Cognitive Psychotherapy, 35, 487-494. Recuperado a partir de https://doi.org/10.1017/S1352465807003761

Kröger, C., Schweiger, U., Sipos, V., Kliem, S., Arnold, R., Schunert, T. \& Reinecker, H. (2010). Dialectical behaviour therapy and an added cognitive behavioural treatment module for eating disorders in women with borderline 
personality disorder and anorexia nervosa or bulimia nervosa who failed to respond to previous treatments: an open trial with a 15 -month followup. Journal of Behavior Therapy and Experimental Psychiatry, 41, 381-388. Recuperado a partir de https://doi.org/10.1016/j.jbtep.2010.04.001

Langhoff, C., Baer, T., Zubraegel, D. \& Linden, M. (2008). Therapist-patient alliance, patient-therapist alliance, mutual therapeutic alliance, therapistpatient concordance, and outcome of CBT in GAD. Journal of Cognitive Psychotherapy: An International Quarterly, 22(1), 68-79. Recuperado a partir de https://doi.org/10.1891/0889.8391.22.1.68

La Rie, S., Noordenbos, G., Donker, M. \& Furth, E. V. (2008). The quality of treatment of Eating Disorders: A comparison of the therapists' and the patients' perspective. International Journal of Eating Disorders, 41(4), 307-317. Recuperado a partir de https://doi.org/10.1002/eat.20494

Leahy, R. L. (2008). The therapeutic relationship in cognitive-behavioral therapy. behavioural and cognitive psychotherapy, 36, 769-777. Recuperado a partir de https://doi.org/10.1017/S1352465808004852

Lieb, K., Zanarini, M. C., Schmahl, C., Linehan, M. M. \& Bohus, M. (2004). Borderline personality disorder. Lancet, 364, 453-461.

Linehan, M. (2010). Terapia cognitivo-comportamental para transtorno da personalidade borderline. Porto Alegre: Artmed.

Lynch, T. R., Chapman, A. L., Rosenthal, Kuo, J. R. \& Linehan, M. M. (2006). Mechanisms of change in dialectical behavior therapy: theoretical and empirical observations. Journal of Clinical Psychology, 62(4), 459-480. Recuperado a partir de https://doi.org/10.1002/jclp.20243

Lynch, T. R. Trost, W. T., Salsman, N., Linehan, M. M. (2007). Dialectical behavior therapy for borderline personality disorder. Clinical Psychology, 3, 181-205. Recuperado a partir de https://doi.org/10.1146/annurev. clinpsy.2.022305.095229

McMain, S., Korman, L. M \& Dimeff, L. (2001). Dialectical behavior therapy and the treatment of emotion dysregulation. Journal of Clinical Psychology, 57(2), 183-196.

McQuillan, A., Nicastro, R., Guenot, F., Girard, M., Lissner, C. \& Ferrero, F. (2005). Intensive dialectical behavior therapy for outpatients with borderline personality disorder who are in crisis. Psychiatric Services, 56(2), 193-197. 
Melo, W. V. (2014). Terapia comportamental dialética. In W. V. Melo (Org.). Estratégias psicoterápicas e a terceira onda em terapia cognitiva. (pp. 314-343). Novo Hamburgo: Sinopsys.

Melo, W. V. \& Fava, D. C. (2012). Oito ou oitenta? In C. B. Neufeld (Org.). Protagonistas em psicoterapia cognitivo-comportamental: histórias de vida e de psicoterapia. (pp. 165-188). Porto Alegre: Sinopsys.

Melo, W. V., Sardinha, A. \& Levitan, M. (2014). O desenvolvimento das terapias cognitivo-comportamentais e a terceira onda. In C. B. Rangé, E. M. O. Falcone \& C. B. Neufeld, (Org.). Programa de atualização em terapia cognitivo-comportamental: Procognitiva. (pp. 9-44). Porto Alegre: Artmed.

Neacsiu, A. D., Rizvi, S. L \& Linehan, M. M. (2010). Dialectical behavior therapy skills use as a mediator and outcome of treatment for borderline personality disorder. Behaviour Research and Therapy, 48(9), 832-839. Recuperado a partir de https://doi.org/10.1016/j.brat.2010.05.017

Scaturo, D. J. (2002). Technical skill and the therapeutic relationship: a fundamental dilemma in cognitive-behavioral and insight-oriented therapy. Family Therapy, 29(1), 1-22.

Thwaites, R. \& Levy, J. B. (2007). Conceptualizing empathy in cognitive behaviour therapy: making the implicit explicit. Behavioural and Cognitive Psychotherapy, 35, 591-612. Recuperado a partir de https://doi.org/10.1017/ S1352465807003785

Trupin, E. W., Steward, D. G., Beach, B. \& Boesky, L. (2002). Effectiveness of a dialectical behaviour therapy program for incarcerated female juvenile offenders. Child and Adolescent Mental Health, 7(3), 121-127. 